\title{
ECM algorithm of Parametric Estimation in constant stress accelerated life for mixture Weibull distribution with type II censoring
}

\author{
Hui Jiang \\ Department Mathematics \\ Qinghai Normal university \\ Xining, China \\ Jh655@163.com
}

\begin{abstract}
Mixed Weibull distributions play an important role in life time data analysis. In this paper, we employ the ECM algorithm to obtain the ML estimation for the mixed Weibull model in the constant stress accelerated life time test with type II censoring. The iteration formulas are given in the paper. Simulations are also made to show the Performance of estimation.
\end{abstract}

Keywords-Mixed Weibull Distribution; EM algorithm; ECM algorithm; censor sample data.

\section{INTRODUCTION}

Life data analysis is a heighted interest problem that is con cerned by statisticians and practical worker of the area on engin eering, medicine, biology and so on. The statistical analysis te chnique to the life of one population is very mature.The life tes ts in constant stress with censer data are popular.

Today's manufactures face strong pressure to develop new, higher technology and longer life products in record time, while improving productivity, product field reliability, and overall quality. This has motivated the development of methods like concurrent engineering and encouraged wider use of designed experiments for product and process improvement.

Accelerated Life Tests (ALT) are used widely in manufacturing industries, particularly to obtain timely information on reliability of simple components and materials. Accelerated Life Test provide information quickly on the life distribution of the materials or products by testing them at higher-than-nominal levels of stress such as high temperature, voltage, pressure, vibration, humidity, cycling rate or load to induce early failures. The results of the accelerated conditions are analyzed in term of a model to relate life length to stress; they are extrapolated to the design stress to estimate the life distribution.

The history of ALT has more than fifty years. Levenbach G J. [1] first presented accelerated life testing of capacitors IRA-trans on reliability and quality control. Bai D S, Kim M S, Lee S L. [2] presented Optimum simple step-stress accelerated life tests with censoring. Nelson W.B[3] studied accelerated life stress models and data analysis. Nelson W B, Hanhn G J.[4] presented linear estimation of a regression relationship from censored data part I: simple methods and their application. Miller \& Nelson [5] described optimum ALT for exponential distribution, and complete data. Shisong Mao [6] presented statistical analysis of step-stress accelerated life test on the exponential distribution. Shisong Mao, Qing Han[7] studied statistical analysis of life and accelerated life test on Weibull distribution case under type I censoring. Zhihua
Zhang [8] studied optimal model of accelerated life test to exponential distribution. But it is few studied on accelerated model of mixed life distributions. Liping zhu, Yiqiang Lu, Shisong Mao [9] studied the accelerated model of mixed exponential distribution. Hui Jiang, Yincai Tang [10,11] studied ECM algorithm of parametric estimation for mixture Weibull distribution and ECM algorithm of parametric estimation in constant stress accelerated life for mixture Weibull distribution. Haifang Yan , Hui Jiang[12,13] presented MCEM accelerated algorithm in constant stress for mixed exponential distribution and MCEM accelerated algorithm in constant stress for mixed exponential distribution under type II censoring .

In this paper, we only considered two Weibull distributions which are mixed and employ the ECM algorithm to obtain the ML estimation for the mixed Weibull model under the constant stress accelerated life time test under type II censoring. The iteration formulas are given in the paper. Simulations are also made to show the performance of estimation.

\section{PARAMETRIC ESTIMATION UNDER TYPE II CENSORING}

We obtain the parametric estimation of mixture Weibull distribution with type II censoring in this section.

$$
\begin{aligned}
& \text { Let } X_{i} \sim \operatorname{Wei}\left(\lambda_{i}, \theta_{i}\right) \text {, its pdf. is } \\
& \qquad \begin{array}{l}
f_{i}\left(x_{i} \mid \lambda_{i}, \theta_{i}\right)=\frac{1}{\lambda_{i}} \theta_{i} x^{\left(\theta_{i}-1\right)} e^{-\frac{1}{\lambda_{i}} x^{\theta_{i}}}, \\
x>0, i=1,2 .
\end{array}
\end{aligned}
$$

If $P\left(X=x_{1}\right)=p, P\left(X=x_{2}\right)=1-p$, we call that $\mathrm{X}$ has mixture Weibull distribution, and its pdf. is

$$
\begin{aligned}
& f(x \mid \varphi)=p \frac{1}{\lambda_{1}} \theta_{1} x^{\left(\theta_{1}-1\right)} e^{-\left(\frac{1}{\lambda_{1}} x^{\theta_{1}}\right)} \\
& +(1-p) \frac{1}{\lambda_{2}} \theta^{2} x^{\left(\theta_{2}-1\right)} e^{-\left(\frac{1}{\lambda_{2}} x^{\theta_{2}}\right)}
\end{aligned}
$$

Where $\varphi=\left(p, \lambda_{1}, \lambda_{2}, \theta_{1}, \theta_{2}\right), 0<p<1, \theta_{1}, \theta_{2}, \lambda_{1}, \lambda_{2}>0$.

\section{A. Assumptions}

1) (Model description) $\mathrm{k}$ accelerated stress levels $S_{1}, S_{2}, \cdots, S_{k}\left(S_{1}<S_{2}<\cdots<S_{k}\right)$ are used. For any level of stress levels $S_{1}, S_{2}, \cdots, S_{k}$, the life distribution of test unit is mixture Weibull. Under $S_{i}$ and constant $p$, the pdf. is 


$$
\begin{aligned}
f_{i}(x) & =p \frac{1}{\lambda_{i 1}} \theta_{i 1} x^{\left(\theta_{i 1}-1\right)} e^{-\left(\frac{1}{\lambda_{i 1}} x^{\theta_{i 1}}\right)} \\
& +(1-p) \frac{1}{\lambda_{i 2}} \theta_{i 2} x^{\left(\theta_{i 2}-1\right)} e^{-\left(\frac{1}{\lambda_{i 2}} x^{\theta_{i 2}}\right)}
\end{aligned}
$$

Where $x>0, i=1,2, \cdots, k$.

2) (Accelerated equation) The shape parametric $\theta_{i}$ has nothing to do with stress level . that is ,

$$
\theta_{1 l}=\theta_{2 l}=\cdots=\theta_{k l}=\theta_{l}, l=1,2 \text {. }
$$

Let $\mu_{i l}=\ln \eta_{i l}, \eta_{i l}=\lambda_{i l}^{1 / \theta_{l}}$, thus $\mu_{i l}$ is a log-linear function of stress. The accelerated equation is,

$$
\ln \mu_{i l}=a_{l}+b_{l} \varphi\left(S_{i}\right), i=0,1, \cdots, k, l=1,2 .
$$

Where $a_{l}, b_{l}$ are unknown parameter, $\varphi\left(S_{i}\right)$ is given function of $S_{i}$. When stress level $\mathrm{S}$ is temperature, $\phi(S)=\frac{1}{S}$. the accelerated equation is called Arrhenins model . When stress level $S$ is electric voltage, $\phi(S)=\ln (S)$. the accelerated equation is called inverse power law model .

3) (The cumulative way) The remaining life of a test unit depends only on the cumulant and stress condition at that time, which is nothing with the cumulative way.( see Miller \& Nelson[5])

\section{B. ECM algorithm}

Suppose $X_{1}, X_{2}, \cdots, X_{r_{i}}$ are preceding $r_{i}$ order statistics from the mixture Weibull distribution (2) with sample size $n$ under stress $S_{i}$ level, which observed values are

$$
x_{1}, x_{2}, \cdots, x_{r_{i}} \cdot\left(r_{1}+r_{2}+\cdots+r_{k}=r_{i}\right)
$$

Note

$$
\begin{aligned}
& f_{1 i j}=f_{1 i j}\left(x_{i j} \mid \varphi_{i}\right)=\frac{\theta_{1}}{\lambda_{1 i}} x_{i j}^{\left(\theta_{1}-1\right)} e^{-\frac{1}{\lambda_{1 i}} x_{i j}^{\theta_{1}}} ; \\
& S_{1 i r_{i}}=S_{1 i r_{i}}\left(x_{i r_{i}} \mid \varphi_{i}\right)=e^{-\frac{x_{i r_{1}^{q_{1}}}}{\lambda_{1 i}}} \text {; } \\
& f_{2 i j}=f_{2 i j}\left(x_{i j} \mid \varphi_{i}\right)=\frac{\theta_{2}}{\lambda_{2 i}} x_{i j}^{\left(\theta_{2}-1\right)} e^{-\frac{1}{\lambda_{2 i}} x_{i j}^{\theta_{2}}} ; \\
& S_{2 i r_{i}}=S_{2 i r_{i}}\left(x_{i r_{i}} \mid \varphi_{i}\right)=e^{-\frac{x_{i i_{i j}}^{\theta_{2}}}{\lambda_{2 i}}} \text {; } \\
& f_{i j}=f_{i j}\left(x_{i j} \mid \varphi_{i}\right)=p f_{1 i j}+(1-p) f_{2 i j} \text {; } \\
& S_{i r_{i}}=S_{i r_{i}}\left(x_{i r_{i}} \mid \varphi_{i}\right)=p s_{1 i r_{i}}+(1-p) S_{2 i r_{i}} \text {. }
\end{aligned}
$$

Where $\varphi_{i}=\left(p, \theta_{1}, \theta_{2}, \eta_{1 i}, \eta_{2 i}\right), i=1,2, \cdots, k, j=1,2, \cdots, n$.
$S_{1 i r_{i}}, S_{2 i r_{i}}$ is survival function.

Use the assumptions given in A, the life distribution of $j$ th product with stress $S_{i}$ is $f_{i j}$.

We give the indicator function $I_{i j}, I_{i j}=1$ express $j$ th product with stress $S_{i}$ from the first Weibull population , $I_{i j}=0$ express $j$ th product with stress $S_{i}$ from the second Weibull population. The joint distribution of $\left(X_{i j}, I_{i j}\right)$ for no censor data is

$$
g\left(x_{i j}, I_{i j}\right)=\left(p f_{1 i j}\right)^{I_{i j}}\left[(1-p) f_{2 i j}\right]^{1-I_{i j}} .
$$

Where $x_{i j} \leq x_{i r_{i}}$. Thus, the conditional probability of the $I_{i j}$ given $X_{i j}$ is

$$
P\left(I_{i j}=1 \mid x_{i j}, \varphi_{i}\right)=\frac{p f_{1 i j}}{f_{i j}} ; P\left(I_{i j}=0 \mid x_{i j}, \varphi_{i}\right)=\frac{(1-p) f_{2 i j}}{f_{i j}}
$$

The joint distribution of $\left(X_{i j}, I_{i j}\right)$ for censor data is

$$
g\left(x_{i r_{i}}, I_{i j}\right)=\left(p s_{1 i j}\right)^{I_{i j}}\left[(1-p) s_{2 i j}\right]^{1-I_{i j}}
$$

Where $x_{i j}>x_{i r_{i}}$.Then, the conditional probability of the $I_{i j}$ given $X_{i j}\left(x_{i j}>x_{i r_{i}}\right)$ is

$$
P\left(I_{i j}=1 \mid x_{i r_{i}}, \varphi_{i}\right)=\frac{p s_{1 i_{i}}}{s_{i r_{i}}} ; P\left(I_{i j}=0 \mid x_{i r_{i}}, \varphi_{i}\right)=\frac{(1-p) s_{2 i r_{i}}}{s_{i r_{i}}} .
$$

The steps of ECM algorithm given initial value $\varphi_{i}^{(0)}$ are

1. (E-step)Evaluating expectation $(\mathrm{m}=1,2, \cdots)$

$$
\begin{aligned}
& Q\left(\varphi_{i}, \varphi_{i}^{(m-1)}\right)=\sum_{i=1}^{k} \sum_{j=1}^{r_{i}} E_{I_{i j} \mid x_{i j}, \varphi_{i}^{(m-1)}}\left[\ln \left(g\left(x_{i j}, I_{i j}, \varphi_{i}\right)\right]\right. \\
& =\sum_{i=1}^{k} \sum_{j=1}^{r_{i}}\left\{c_{1 i j}^{(m-1)} \ln \left(p f_{1 i j}\right)+c_{2 i j}^{(m-1)} \ln \left[(1-p) f_{2 i j}\right]\right\} \\
& +\sum_{i=1}^{k}\left(n-r_{i}\right)\left\{d_{1 i r_{i}}^{(m-1)} \ln \left(p s_{1 i r_{i}}\right)+d_{2 i r_{i}}^{(m-1)} \ln \left[(1-p) s_{1 i r_{i}}\right]\right\} \\
& =\sum_{i=1}^{k} \sum_{j=1}^{r_{i}}\left\{c_{1 i j}^{(m-1)}\left[\ln p-\ln \lambda_{1 i}+\ln \theta_{1}+\left(\theta_{1}-1\right) \ln x_{i j}-\frac{1}{\lambda_{1 i}} x_{i j}^{\theta_{1}}\right]\right. \\
& \left.+c_{2 i j}^{(m-1)}\left[\ln (1-p)-\ln \lambda_{2 i}+\ln \theta_{2}+\left(\theta_{2}-1\right) \ln x_{i j}-\frac{1}{\lambda_{2 i}} x_{i j}^{\theta_{2}}\right]\right\} \\
& +\sum_{i=1}^{k}\left\{\left(n-r_{i}\right)\left[d_{1 i r_{i}}^{(m-1)}\left(\ln p-\frac{x_{1 r_{i}}^{\theta_{1}}}{\lambda_{1 i}}\right)+d_{2 i r_{i}}^{(m-1)}\left(\ln (1-p)-\frac{x_{1 r_{i}}^{\theta_{2}}}{\lambda_{2 i}}\right]\right\}\right.
\end{aligned}
$$


Where

$$
\begin{aligned}
& c_{1 i j}^{(m-1)}=\frac{p^{(m-1)} f_{1 i j}^{(m-1)}}{f_{i j}^{(m-1)}}, \quad c_{2 i j}^{(m-1)}=\frac{\left(1-p^{(m-1)}\right) f_{2 i j}^{(m-1)}}{f_{i j}^{(m-1)}} ; \\
& d_{1 i j}^{(m-1)}=\frac{p^{(m-1)} s_{1 i r_{i}}^{(m-1)}}{s_{i r_{i}}^{(m-1)}}, \quad d_{2 i j}^{(m-1)}=\frac{\left(1-p^{(m-1)}\right) s_{2 i r_{i}}^{(m-1)}}{s_{i r_{i}}^{(m-1)}} ; \\
& f_{1 i j}^{(m-1)}=f_{1 i j}\left(x_{i j} \mid \varphi^{(m-1)}\right), \\
& s_{1 i r_{i}}^{(m-1)}=s_{1 i j}\left(x_{i j} \mid \varphi^{(m-1)}\right), \quad s_{2 i j}^{(m-1)}=f_{2 i j}\left(x_{i j} \mid \varphi^{(m-1)}\right) ; \\
& \varphi_{i}^{(m-1)}=\left(p^{(m-1)}, \lambda_{1 i}^{(m-1)}, \lambda_{2 i}^{(m-1)}, \theta_{1}^{(m-1)}, \theta_{2}^{(m-1)}\right) .
\end{aligned}
$$

2. (M-step) Maximization, find $\varphi_{i}^{(m)}$ which let

$$
Q\left(\varphi_{i}^{(m)}, \varphi_{i}^{(m-1)}\right)=\max Q\left(\varphi_{i}, \varphi_{i}^{(m-1)}\right) .
$$

3. Take $\varphi_{i}^{(m)}$ as the renewal value of $\varphi_{i}^{(m-1)}$. Repeat the first step and the second step and stop iteration when $\left\|\varphi_{i}^{(m)}-\varphi_{i}^{(m-1)}\right\|$ is lesser than a given threshold. This iteration process must be convergence due to the convergence of ECM algorithm get guarantee in theory. But the ECM algorithm may converge to the local optima value. It is suggestion that more than one different initial value are selected to iteration, then judge the convergence by comparing the likelihood function on extreme values. After that we get the parametric estimation.

In this paper, it is hard to obtain $\varphi_{i}^{(m)}$ because the equation of vector $\varphi_{i}$ is transcendental equation.we obtain the solution by ECM algorithm and Newton iteration. ECM algorithm is special EM algorithm that is process of $\mathrm{k}$-step conditional maximization in EM algorithm. Suppose $\varphi_{i}=\left(\varphi_{i 1}, \varphi_{i 1}, \cdots, \varphi_{i k}\right)$, and let $\varphi_{i}^{(m-1)}=\left(\varphi_{i 1}^{(m-1)}, \varphi_{i 2}^{(m-1)}, \cdots \varphi_{i k}^{(m-1)}\right)$, then we obtain $\varphi_{i 1}$ which maximize $Q\left(\varphi_{i}, \varphi_{i}^{(m-1)}\right)$ on given $\varphi_{i 2}=\varphi_{i 2}^{(m-1)}, \cdots, \varphi_{i k}=\varphi_{i k}^{(m-1)}$ in m-step iteration. Let $\varphi_{i 1}=\varphi_{i 1}^{(m)}$. Then get $\varphi_{i 2}$ which maximize $Q\left(\varphi_{i}, \varphi_{i}^{(m-1)}\right)$ on given $\varphi_{i 1}=\varphi_{i 1}^{(m)}, \varphi_{i j}=\varphi_{i j}^{(m-1)}$ $j=3, \cdots, k$. Let $\varphi_{i 2}=\varphi_{i 2}^{(m)}$ and go on like this way, we get $\varphi_{i}^{(m)}=\left(\varphi_{i 1}^{(m)}, \varphi_{i 2}^{(m)}, \cdots, \varphi_{i k}^{(m)}\right)$ and finish one iteration. In this paper, $k=5$.

First, let

$$
\frac{\partial Q\left(\varphi_{i}, \varphi_{i}^{(m-1)}\right)}{\partial p}=0
$$

We obtain

$$
p^{(m)}=\frac{\sum_{i=1}^{k}\left[\sum_{j=1}^{r_{i}} c_{1 i j}^{(m-1)}+\left(n-r_{i}\right) d_{1 i_{i}}^{(m-1)}\right]}{\sum_{i=1}^{k}\left[\sum_{j=1}^{r_{i}}\left(c_{1 i j}^{(m-1)}-c_{2 i j}^{(m-1)}+\left(n-r_{i}\right)\left(d_{1 i r_{i}}^{(m-1)}-d_{2 i r_{i}}^{(m-1)}\right)\right]\right.} .
$$

Second, let

$$
\left.\frac{\partial Q\left(\varphi_{i}, \varphi_{i}^{(m-1)}\right)}{\partial \lambda_{l i}}\right|_{\varphi_{i}^{(m-1)}}=0,(l=1,2 .)
$$

We obtain

$$
\lambda_{1 i}=\frac{\sum_{j=1}^{r_{i}} c_{1 i j}^{(m-1)} x_{i j}^{\theta_{l}}+\left(n-r_{i}\right) d_{1 i r_{i}}^{(m-1)} x_{i r_{i}}^{\theta_{l}}}{\sum_{j=1}^{r_{i}} c_{1 i j}^{(m-1)}},
$$

Where $\varphi_{i}^{(m-1)}=\left(p^{(m)}, \lambda_{1 i}^{(m-1)}, \lambda_{2 i}^{(m-1)}, \theta_{1}^{(m-1)}, \theta_{2}^{(m-1)}\right)$.

In same way, We obtain

$$
\lambda_{2 i}=\frac{\sum_{j=1}^{r_{i}} c_{2 i j}^{(m-1)} x_{i j}^{\theta_{l}}+\left(n-r_{i}\right) d_{2 i r_{i}}^{(m-1)} x_{i r_{i}}^{\theta_{l}}}{\sum_{j=1}^{r_{i}} c_{2 i j}^{(m-1)}},
$$

Where $\varphi_{i}^{(m-1)}=\left(p^{(m)}, \lambda_{1 i}^{(m)}, \lambda_{2 i}^{(m-1)}, \theta_{1}^{(m-1)}, \theta_{2}^{(m-1)}\right)$.

Last, let

$$
\frac{\partial Q\left(\varphi_{i}, \varphi_{i}^{(m-1)}\right)}{\partial \theta_{l}}=0, \quad(l=1,2 .)
$$

Then we get equation

$$
\begin{aligned}
& \sum_{i=1}^{k} \sum_{j=1}^{r_{i}} c_{l i j}^{(m-1)}+\theta_{l} \sum_{i=1}^{k} \sum_{j=1}^{r_{i}} c_{l i j}^{(m-1)} \ln x_{i j} \\
& -\sum_{i=1}^{k}\left[\sum_{j=1}^{r_{i}} c_{l i j}^{(m-1)} x_{i j}^{\theta_{l}} \ln x_{i j}+\sum_{i=1}^{k}\left(n-r_{i}\right) d_{l i r_{i}}^{(m-1)} x_{i r_{i}}^{\theta_{l}} \ln x_{i r_{i}}\right] \frac{\theta_{l}}{\lambda_{l i}}=0 .
\end{aligned}
$$

We obtain the solution of (9) by using Newton iteration method. Let

$$
\begin{aligned}
& f\left(\theta_{l}\right)=\sum_{i=1}^{k} \sum_{j=1}^{r_{i}} c_{l i j}^{(m-1)}+\theta_{l} \sum_{i=1}^{k} \sum_{j=1}^{r_{i}} c_{l i j}^{(m-1)} \ln x_{i j} \\
& -\left[\sum_{i=1}^{k} \sum_{j=1}^{r_{i}} \frac{1}{\lambda_{l i}} c_{l i j}^{(m-1)} x_{i j}^{\theta_{l}} \ln x_{i j}+\sum_{i=1}^{k} \frac{1}{\lambda_{l i}}\left(n-r_{i}\right) d_{l i r_{i}}^{(m-1)} x_{i r_{i}}^{\theta_{l}} \ln x_{i r_{i}}\right] \theta_{l}
\end{aligned}
$$

Then

$$
\begin{aligned}
& \frac{\partial f\left(\theta_{l}\right)}{\partial \theta_{l}}=\sum_{i=1}^{k} \sum_{j=1}^{r_{i}} c_{l i j}^{(m-1)} \ln x_{i j}-\sum_{i=1}^{k} \sum_{j=1}^{r_{i}} \frac{1}{\lambda_{l i}} c_{l i j}^{(m-1)} x_{i j}^{\theta_{l}} \ln x_{i j} \\
& -\sum_{i=1}^{k} \frac{1}{\lambda_{l i}}\left(n-r_{i}\right) d_{l i r_{i}}^{(m-1)} x_{i r_{i}}^{\theta_{l}} \ln x_{i r_{i}}-\theta_{l} \sum_{i=1}^{k} \sum_{j=1}^{r_{i}} \frac{1}{\lambda_{l i}} c_{l i j}^{(m-1)} x_{i j}^{\theta_{l}} \ln ^{2} x_{i j} \\
& -\theta_{l} \sum_{i=1}^{k} \frac{1}{\lambda_{l i}}\left(n-r_{i}\right) d_{l i r_{i}}^{(m-1)} x_{i r_{i}}^{\theta_{l}} \ln ^{2} x_{i r_{i}} \\
& \quad \text { Note }(l=1,2 .)
\end{aligned}
$$




$$
\begin{aligned}
& A_{l}\left(x_{i j}\right)=\sum_{i=1}^{k} \sum_{j=1}^{r_{i}} c_{l i j}^{(m-1)} \ln x_{i j} ; \\
& B_{l}\left(x_{i j}, \lambda_{l i}\right)=\sum_{i=1}^{k} \sum_{j=1}^{r_{i}} \frac{1}{\lambda_{l i}} c_{l i j}^{(m-1)} x_{i j}^{\theta_{l}} \ln x_{i j} ; \\
& C_{l}\left(x_{i j}, \lambda_{l i}\right)=\sum_{i=1}^{k} \frac{1}{\lambda_{l i}}\left(n-r_{i}\right) d_{l i r_{i}}^{(m-1)} x_{i r_{i}}^{\theta_{l}} \ln x_{i r_{i}} ; \\
& D_{l}\left(x_{i j}, \lambda_{l i}\right)=\sum_{i=1}^{k} \sum_{j=1}^{r_{i}} \frac{1}{\lambda_{l i}} c_{l i j}^{(m-1)} x_{l i}^{\theta_{l}} \ln ^{2} x_{i j} ; \\
& E_{l}\left(x_{i j}, \lambda_{l i}\right)=\sum_{i=1}^{k} \frac{1}{\lambda_{l i}}\left(n-r_{i}\right) d_{l i r_{i}}^{(m-1)} x_{i r_{i}}^{\theta_{l}} \ln ^{2} x_{i r_{i}} ; \\
& F_{l}=\sum_{i=1}^{k} \sum_{j=1}^{r_{i}} c_{l i j}^{(m-1)} .
\end{aligned}
$$

Thus, the solution of (8) is

$$
\theta_{l}^{(m)}=\theta_{l}^{(m-1)}-\left.f\left(\theta_{l}^{(m-1)}\right)\left[\frac{\partial f\left(\theta_{l}\right)}{\partial \theta_{l}}\right]^{-1}\right|_{\theta_{l}=\theta_{l}^{(m-1)}} .
$$

This is

$$
\begin{aligned}
& \theta_{l}^{(m)}=\theta_{l}^{(m-1)} \\
& -\frac{\left[A_{l}\left(x_{i j}\right)-B_{l}\left(x_{i j}, \lambda_{l i}\right)-C_{l}\left(x_{i j}, \lambda_{l i}\right)\right] \theta_{l}+F_{l}}{A_{l}\left(x_{i j}\right)-B_{l}\left(x_{i j}, \lambda_{l i}\right)-C_{l}\left(x_{i j}, \lambda_{l i}\right)-\left[D_{l}\left(x_{i j}, \lambda_{l i}\right)+E_{l}\left(x_{i j}, \lambda_{l i}\right)\right] \theta_{l}} .
\end{aligned}
$$

When $l=1$ in(8),

$$
\varphi_{i}^{(m-1)}=\left(p^{(m)}, \lambda_{1 i}^{(m)}, \lambda_{2 i}^{(m)}, \theta_{1}^{(m-1)}, \theta_{2}^{(m-1)}\right) .
$$

When $l=2$ in (8),

$$
\varphi_{i}^{(m-1)}=\left(p^{(m)}, \lambda_{1 i}^{(m)}, \lambda_{2 i}^{(m)}, \theta_{1}^{(m)}, \theta_{2}^{(m-1)}\right) .
$$

\section{Simulation Analysis}

We choose true value of paramete r $p=0.6, \theta_{1}=6.5$, $\theta_{2}=1.5, \lambda_{11}=4.0, \lambda_{12}=2.0, \lambda_{21}=1.0, \lambda_{22}=0.5$, and repeat 1000 tests on sample size $50,60,80,100$ for verifying the efficiency of ECM algorithm on estimating parameter of Weibull distribution. The simulation initial values are $p=0.5, \theta_{1}=7.0$

\begin{tabular}{|c|c|c|c|c|c|}
\hline & & $n=50$ & $\mathrm{n}=60$ & $\mathrm{n}=80$ & $\mathrm{n}=100$ \\
\hline$\hat{P}$ & $\begin{array}{c}\text { MEAN } \\
\text { MES }\end{array}$ & $\begin{array}{l}5.91 \mathrm{E}-01 \\
3.92 \mathrm{E}-02\end{array}$ & $\begin{array}{l}5.91 \mathrm{E}-01 \\
3.19 \mathrm{E}-02\end{array}$ & $\begin{array}{c}5.91 \mathrm{E}-02 \\
3.2 \mathrm{E}-02\end{array}$ & $\begin{array}{l}5.93 \mathrm{E}-01 \\
1.73 \mathrm{E}-02\end{array}$ \\
\hline$\hat{\theta}_{1}$ & $\begin{array}{c}\text { MEAN } \\
\text { MES }\end{array}$ & $\begin{array}{c}7.34 \mathrm{E} 00 \\
1.54 \mathrm{E}-01\end{array}$ & $\begin{array}{c}7.21 \mathrm{E} 00 \\
1.07 \mathrm{E}-01\end{array}$ & $\begin{array}{c}6.98 \mathrm{E} 00 \\
6.77 \mathrm{E}-02\end{array}$ & $\begin{array}{c}6.87 \mathrm{E} 00 \\
3.40 \mathrm{E}-02\end{array}$ \\
\hline$\hat{\theta}_{2}$ & $\begin{array}{c}\text { MEAN } \\
\text { MES }\end{array}$ & $\begin{array}{c}1.60 \mathrm{E} 00 \\
6.42 \mathrm{E}-02\end{array}$ & $\begin{array}{c}1.58 \mathrm{E} 00 \\
4.31 \mathrm{E}-02\end{array}$ & $\begin{array}{c}1.56 \mathrm{E} 00 \\
2.94 \mathrm{E}-02\end{array}$ & $\begin{array}{r}1.54 \mathrm{E} 00 \\
1.81 \mathrm{E}-02\end{array}$ \\
\hline$\hat{\lambda}_{11}$ & $\begin{array}{c}\text { MEAN } \\
\text { MES }\end{array}$ & $\begin{array}{l}9.53 \mathrm{E} 02 \\
5.22 \mathrm{E} 07\end{array}$ & $\begin{array}{l}9.36 \mathrm{E} 00 \\
7.13 \mathrm{E} 02\end{array}$ & $\begin{array}{l}5.19 \mathrm{E} 00 \\
5.45 \mathrm{E} 00\end{array}$ & $\begin{array}{c}4.64 \mathrm{E} 00 \\
2.50 \mathrm{E}-01\end{array}$ \\
\hline$\hat{\lambda}_{12}$ & $\begin{array}{c}\text { MEAN } \\
\text { MES }\end{array}$ & $\begin{array}{l}1.43 \mathrm{E} 00 \\
1.36 \mathrm{E} 01\end{array}$ & $\begin{array}{c}1.19 \mathrm{E} 00 \\
1.85 \mathrm{E}-01\end{array}$ & $\begin{array}{l}1.46 \mathrm{E} 00 \\
1.77 \mathrm{E} 01\end{array}$ & $\begin{array}{c}1.17 \mathrm{E} 00 \\
1.82 \mathrm{E}-01\end{array}$ \\
\hline$\hat{\lambda}_{21}$ & $\begin{array}{c}\text { MEAN } \\
\text { MES }\end{array}$ & $\begin{array}{c}1.10 \mathrm{E} 00 \\
2.11 \mathrm{E}-01\end{array}$ & $\begin{array}{c}1.10 \mathrm{E} 00 \\
5.71 \mathrm{E}-01\end{array}$ & $\begin{array}{c}1.05 \mathrm{E} 00 \\
9.12 \mathrm{E}-02\end{array}$ & $\begin{array}{c}1.04 \mathrm{E} 00 \\
4.60 \mathrm{E}-02\end{array}$ \\
\hline$\hat{\lambda}_{22}$ & $\begin{array}{c}\text { MEAN } \\
\text { MES }\end{array}$ & $\begin{array}{l}1.06 \mathrm{E} 00 \\
1.55 \mathrm{E} 01\end{array}$ & $\begin{array}{l}1.06 \mathrm{E} 00 \\
1.71 \mathrm{E} 02\end{array}$ & $\begin{array}{l}8.55 \mathrm{E}-01 \\
6.31 \mathrm{E}-01\end{array}$ & $\begin{array}{l}8.49 \mathrm{E}-01 \\
5.78 \mathrm{E}-01\end{array}$ \\
\hline
\end{tabular}

$\theta_{2}=2, \quad \lambda_{11}=4.5, \quad \lambda_{12}=2.5, \lambda_{21}=1.5, \lambda_{22}=0.9$.Sample mean and mean square error of parameters are given in table 1 . the result show ECM algorithm that estimate parameter of mixture(mixed) Weibull distribution is feasible for medium sample size $(n>50)$ and the precision is higher as increasing sample size. But its efficiency is lower when sample size is small, because it is difficult to draw a distinction in two distributions with small sample size.
TABLE 1 Estimation of parameters with type II censoring.(Truncation ratio is $40 \%$ )

\section{REFERENCES}

[1] Levenbach GJ. Accelerated life testing of capacitors IRA-trans on reliability and quality control[J]. PGRQC, 1957,10(1):p9-20

[2] Bai D S, Kim M S, Lee S L. Optimum simple step-stress accelerated life tests with censoring[J]. IEEE trans on reliability,1989,38(5):p528-531.

[3] Nelson WB. Accelerated life stress models and data analysis[J]. IEEE trans on reliability,1980,29(2):p103-108.

[4] Nelson WB, Hahn GJ. Linear estimation of a Regression relationship From censored data part I: Simple Methods and Their Application[J]. Technometrics.1972,14(1):p247-267.

[5] Miller R, Nelson WB. Optimum simple step-stress plans for accelerated life testing [J]. IEEE trans on reliability, 1983, 32(1)p59-65.

[6] Shisong Mao. Statistical analysis of step-stress accelerated life test on the exponential distribution[J]. Acta Mathematicae Applicateae Sinica, 1985, 5(2) p311-316.

[7] Shisong Mao, Qing Han. Statistical analysis of life and accelerated life test on weibull distribution case under type I censoring[J]. Chinese Journal of Applied Probability and Statistics,1991,7(1):p61-72.

[8] Zhihua Zhang. Optimum ALT plans for exponential distribution[J]. Chinese Journal of Applied Probability and Statistics,2001,17(2):p303307.

[9] Liping Zhu, Yiqiang Lu, Shisong Mao. Parametric estimation of mixed exponential distribution [J]. Chinese Journal of Applied Probability and Statistics, 2006, 5(22)p138-149.

[10] Hui Jiang, Yingcai Tong. Estimation of parameters of mixed weibull distributions[J].Sys. Sci. \& Math. Scis. 30(1) $(2010,1)$ p79 - 88.

[11] Hui Jiang, Mei Liu, Yingcai Tong. ECM algorithm of Parametric Estimation in constant stress accelerated life for mixture weibull Distribution. 2011 International Symposium on Statistics \& Manage -ment Science, 2011p21- 24.

[12] Haifang Yan, Hui Jiang, MCEM accelerated algorithm in constant stress for mixture exponential distribution with data censoring[J]. Mathemati -csin Practice and Theory,2012,42(1)p127-134.

[13] Haifang Yan, Hui Jiang, MCEM algorithm of parameters estimation in mixture exponential distribution for constant stress accelerated[J], Natural Science Journal of Xiangtan University,2011,33(3)p35-37,60. 